\title{
The Notch1 gene may control cell chemoresistance in esophageal squamous cell cancer
}

\author{
Kun Gao, Wenqun Xing, Xianben Liu, Jizhao Liu, Haibo Sun, Wentao Hao, Yan Zheng \\ Department of Thoracic Surgery, The Affiliated Cancer Hospital of Zhengzhou University, Henan Cancer Hospital, Zhengzhou, China \\ Contributions: (I) Conception and design: Y Zheng, K Gao; (II) Administrative support: W Xing, X Liu; (III) Provision of study materials or patients: \\ W Xing, X Liu, Y Zheng, H Sun; (IV) Collection and assembly of data: K Gao, W Hao, J Liu; (V) Data analysis and interpretation: K Gao, Y Zheng, \\ W Hao; (VI) Manuscript writing: All authors; (VII) Final approval of manuscript: All authors. \\ Correspondence to: Yan Zheng, MD, PhD. Department of Thoracic Surgery, The Affiliated Cancer Hospital of Zhengzhou University, Henan Cancer \\ Hospital, Zhengzhou 450008, China. Email: zlyysunnyzheng@zzu.edu.cn.
}

\begin{abstract}
Background: In our previous study, missense mutations in the Notch1 gene were found in chemotherapyresistant esophageal squamous cell cancer (ESCC) patients. In this study, we explored changes in the interaction between Notch1 and DLL4 resulting from missense mutations.

Methods: Bioinformatics analysis was performed to assess and compare the different biological structures and functions of wild type (WT) and mutation type (MT) sequences of Notch1. A genetic information search was performed, and the results were analyzed using in silico modeling. Homology modeling of the Notch1 protein was carried out using Swiss-Model software, and modeling of site-directed mutations was carried out using PyMOL software to observe the protein structure. The Notch1-DLL4 ligand-receptor complex protein model was constructed, Wincoot software was used to determine site-directed mutations, and a protein-ligand interaction profiler (PLIP) was used to calculate the noncovalent interactions in the complex.

Results: The mutation site was located in the region where Notch1 binds to DLL4. A careful examination of the in silico structural model revealed that the mutation caused an alteration in the surface charge, and the water-bridge bonds of the interaction between Notch1-DLL4 increased in number from 5 to 7 .

Conclusions: Notch1 gene missense mutation leads to an increase in the number of water-bridge bonds, thus enhancing the Notch1-DLL4 interaction, which may lead to tighter Notch1-DLL4 binding, either making the pathway easier to activate or increasing the length of time it is active.
\end{abstract}

Keywords: Notch1; esophageal cancer; chemoresistance; missense mutation; in silico modeling

Submitted Mar 12, 2021. Accepted for publication Jun 04, 2021.

doi: $10.21037 /$ tcr-21-447

View this article at: https://dx.doi.org/10.21037/tcr-21-447

\section{Introduction}

Neoadjuvant chemotherapy (NAC) combined with surgery has become the standard treatment for patients with locally advanced esophageal cancer in Japan, based on the results of the Japan Clinical Oncology Group Trial 9907 (JCOG9907) (1). However, the results of our previous meta-analysis showed that nearly $40 \%$ of patients did not benefit from NAC, including approximately $5 \%$ of patients who experienced disease progression (2). Therefore, the key to the treatment of esophageal cancer lies in the screening of patients who will potentially benefit from chemotherapy and the adoption of new treatments for those who are resistant to chemotherapy. Nevertheless, the mechanism of NAC resistance in esophageal squamous cell carcinoma (ESCC) is not well understood thus far, and only some studies have demonstrated that the overexpression of certain genes may be related to NAC resistance. For example, the overexpression of MUC4 and MUC20 (3); CDKN1A, ELAVL2 and TSPAN4 (4); and $\triangle \mathrm{Np} 63$ and SOX2 (5). The overexpression of these genes may serve as a biomarker to indicate resistance to NAC; however, evaluation at the 
gene expression level is a complex and error-prone process. Fortunately, with the maturation of next-generation sequencing (NGS) technology, gene sequencing has shown great advantages in accuracy compared to gene protein expression analysis because genes are immutable factors, making them easier to explore than proteins.

In a previous study, we performed NGS on patients with esophageal cancer after NAC and compared them with the sequencing results of samples before chemotherapy. Ultimately, we discovered that the NM_017617.3:c.1348G>A (p. Glu450 Lys) mutation of the NOTCH1 gene was likely to be related to NAC resistance in esophageal cancer (6). Currently, 4 Notch receptors (Notch1-4) and 5 ligands (DLL1, 3, 4 and JAG1, 2) have been identified in mammals $(7,8)$. The interaction between these receptors and ligands controls the differentiation, proliferation, and apoptosis events of mammalian tissues. The Notch1 signaling pathway is a current focus area in tumor research. Existing studies have shown that the Notch1 signaling pathway is overactivated due to gainof-function (GoF) Notch1 alterations in leukemia (9-12), non-small-cell lung cancer (NSCLC) (13), and breast cancer (14). However, the Notch1 signaling pathway is complex in ESCC, showing both under- and overexpression $(15,16)$. Notch 1 epidermal growth factor-like repeat sequence 11 interacts with the DLL4 Delta/Serrate/lag-2 (DSL) domain, and the 450th amino acid of the mutation is located in the Notch1 EGF-like repeat sequence 11 (17). Therefore, the change in the 450th amino acid may affect the interaction between Notch1 and DLL4, which may be related to NAC resistance in patients with ESCC.

In this study, we analyzed the changes in the interaction between Notch1 and DLL4 by constructing protein models of the Notch1 receptor and ligand to speculate on the role of mutated genes in NAC resistance. We expected to determine the molecular mechanism of NAC resistance in patients with ESCC to screen suitable patients for NAC and avoid toxicity in patients with drug resistance.

\section{Methods}

The study was conducted in accordance with the Declaration of Helsinki (as revised in 2013).

\section{Query and analyze Notch1 genetic information}

Notch 1 genetic information was obtained from the UCSC Genome Browser. Information about the
NM_017617.3:c.1348G>A mutation site was acquired according to the naming rules of the mutation site formulated by the Human Genome Variation Society (HGVS). We used Clustalw software to analyze the results of multiple sequence alignment of the Notch1 protein from 12 species, including humans, chimpanzees, macaques, dogs, cows, mice, rats, chickens, zebrafish, Drosophila, anopheles, and frogs.

\section{In silico analyses: molecular structure simulation of buman Notch1 protein}

We used Swiss-Model software to conduct homologous modeling of the human Notch1 protein. In the results, we found that model $5 \mathrm{UK} 5.1$. A represented the mutation site E450 (coverage was 298-488 AA), and the sequence identity reached $96.91 \%$, including the EGF-like domain (412-450 AA) in the covered sequence. Then, we used PyMOL software to analyze the $3 \mathrm{D}$ structure of the missense mutation of the Notch 1 protein and carried out site-directed mutagenesis.

\section{In silico analyses: interaction analysis between Notch1 and DLL4}

The crystal structure model (4xl1.pdb) of the interaction between Notch 1 and DLL4 in Rattus norvegicus was obtained from the PDB database, and the comparative analysis of the Notch1 protein in Rattus norvegicus and humans revealed that the sequence identity was $90.61 \%$. This model (4xl1.pdb) was used to construct the protein interaction model because of the high sequence similarity, and Wincoot software was used for site-directed mutations. A protein-ligand interaction profiler (PLIP) was used to calculate the noncovalent interactions in the Notch1-DLL4 complex before and after mutation to predict the impact of mutations on protein interactions (Figure 1).

\section{Results}

\section{Notcb1 genetic information}

The human Notch 1 gene is located on band 3 of region 34 of the long arm of chromosome 9 and consists of 34 exons and 33 introns. The Notch 1 protein consists of a total of 2,555 amino acid residues. The transcript NM_017617.3 is $9309 \mathrm{bp}$ in length, and the coding sequence (CDS) length is 7,668 bp $[1-7,668]$. The mutation site is located in exon no. 


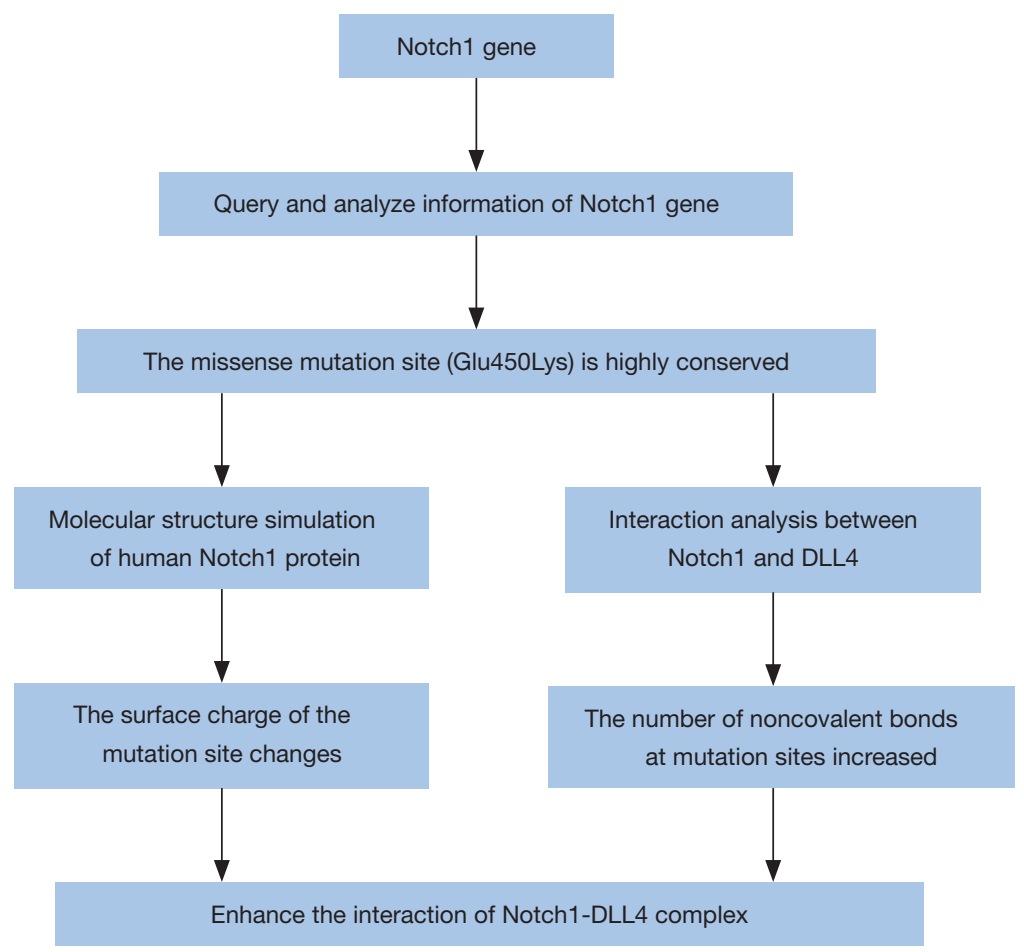

Figure 1 Flowchart of the study. To analyze the surface charge change of the Notch1 protein, we first conducted homology modeling by Swiss-Model software and then gene mutation analysis by PyMOL software. To analyze the changes in the interaction forces of Notch1DLL4, we found the interaction model of Notch1-DLL4 in Rattus norvegicus, carried out gene mutation modeling by Wincoot software, and used PLIP to calculate the interaction force of Notch1-DLL4. PLIP, protein-ligand interaction profiler.

8, and the mutation from $\mathrm{G}$ to A results in the 450th amino acid of the peptide changing from Glu (E) to Lys (K). The mutation site (E450) is located in the epidermal growth factor-like domain (EGF_CA) from the Notch1 conserved domain in humans, and the interval is $412-450 \mathrm{AA}$. Multiple sequence alignment analysis showed that glutamic acid located at position 450 of the Notch1 protein is highly conserved (Figure 2 red box and black characters).

\section{The alteration of the surface potential of Notch1}

The effect of the amino acid mutation (Glu450Lys) on the protein structure was observed after a site-directed mutation, which resulted in a change in the surface charge from negative to positive at the 450th amino acid (Figure 3). E450 was located in the EGF domain of the contact interface between Notch1 and DLL4 proteins, and its mutation caused changes in the surface charge, which might affect the binding of the Notch1 protein to DLL4.

\section{The change in the interaction force between Notch1 and DLL4}

As shown in Figure $4 A$, there were two water bridges and two hydrogen bonds formed by the residues of GLU-424, ARG-448 and GLU-450 in the A chain (Notch1) near the amino acid at the 450th position, together with TRY198 and TRY196 of DLL4. However, as shown in Figure 4B, the Notch1 protein was deformed slightly due to the mutations, and there were three water bridges and two hydrogen bonds formed by the residues of GLU-424, ARG-448 and GLU-450 in the A chain (Notch1) near the amino acid at the 450th position, together with TRY198 and TRY196 of DLL4.

As shown in Figure $4 C$, there were three water bridges, three hydrogen bonds, two hydrophobic bonds, and one pi bond formed by the residues of GLU-424, ARG-448, and GLU-450 in the A chain (Notch1) near the amino acid at the 450th position, together with ARG191, LEU187, 


1. Humans
2. Chimpanzees
3. Macaques
4. Dogs
5. Cows
6. Mice
7. Rats
8. Chickens
9. Zebrafish
10. Drosophila
11. Anopheles
12. Frogs

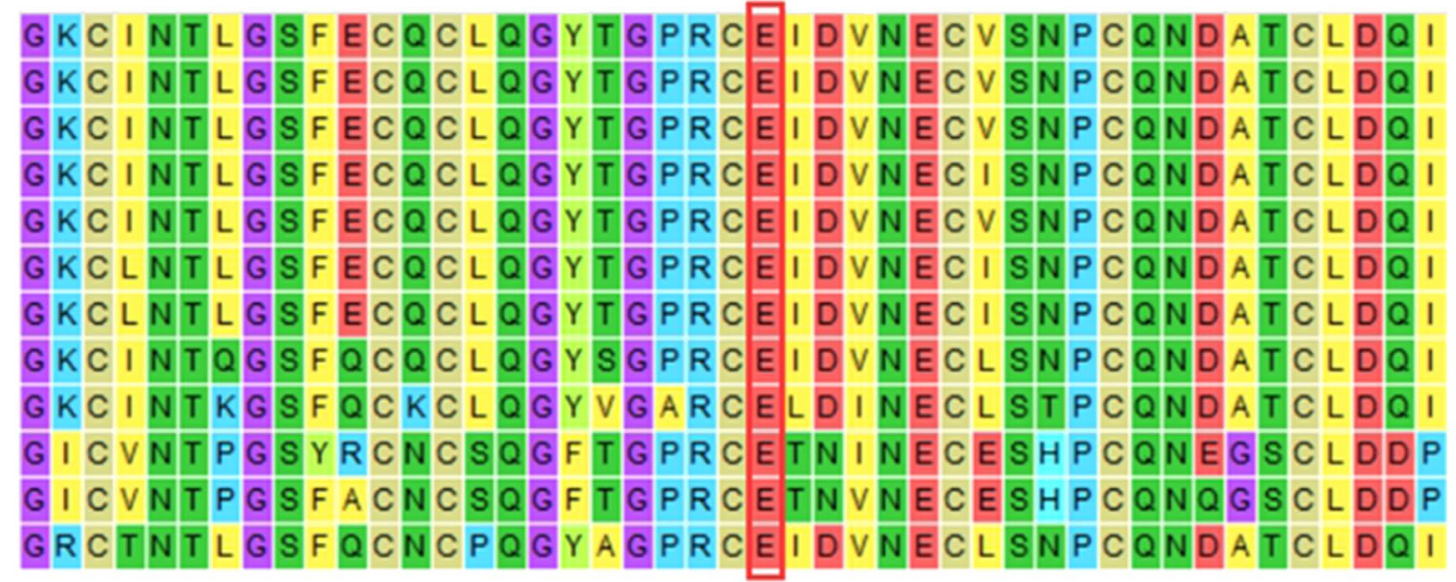

Figure 2 Results of multiple sequence alignment. The red frame is a mutant amino acid, and multiple sequence alignment of the partial amino acid sequence of the NOTCH1 protein showed conservation of glutamic acid 450 across different species.

TRY198, and TRY216 of DLL4. However, as shown in Figure $4 D$, there were four water bridges, three hydrogen bonds, two hydrophobic bonds, and one pi bond formed by the residues of GLU-424, ARG-448, and GLU-450 in the A chain (Notch1) near the amino acid at the 450th position, together with ARG191, LEU187, TRY198, and TRY216 of DLL4 (Figure 4).

The mutation resulted in the addition of two water bridges to the Notch1-DLL4 complex compared with the number of water bridges before the mutation, resulting in an enhanced interaction between the mutant Notch1 and DLL4 near the amino acid residues at position 450, which might increase the overall stability of the protein (Table 1).

\section{Discussion}

ESCC is a complex disease involving multiple genes and factors related to heredity. It can be distributed or inherited in families. The Notch gene was first discovered in fruit flies in 1919, and it was named for causing "notches" in the wing margin in flies (18). The Notch receptor mainly consists of an extracellular region, a transmembrane region and an intracellular region, and it is expressed as a heterodimeric protein on the cell surface. The structural domain of the extracellular portion consists of 29-36 epidermal growth factor (EGF)-like repeats and 3 cysteinerich Lin Notch repeats (LNRs). The primary function of these domains is to bind ligands and initiate Notch. The Notch intracellular domain (NICD) consists of the following five parts: a RAM region, which can bind to DNA binding proteins; six ankyrin repeats (ANK), acting as an enhancer of Notch, mediating the interaction between Notch and other proteins; two nuclear localization signals (NLS); a transactivation domain (TAD), which has not yet been defined for Notch3 and Notch4; and a PEST (praline-glutamate-serine-threonine-rich domain) region, which is related to the stability of the Notch receptor (19). Notch signaling controls mammalian tissue differentiation, proliferation, and apoptosis events. Abnormal Notch signaling, including GoF Notch1 alterations, is involved in the biological processes of a several tumors, such as chronic lymphocytic leukemia, T-ALL, diffuse large B cell lymphoma, mantle cell lymphoma, NSCLC, and breast cancer (9-14) and LoF Notch1 alterations are found in tumor such as HNSCC, cutaneous squamous cell carcinoma, and SCLC (20-23). The role of Notch1 signaling in ESCC was not studied until relatively recently, and its role is not yet clear. A study of esophageal tissue in 9 patients showed that the Notch 1 mutation was several times more common in the normal esophagus than in esophageal cancer (15), and another study focused on the exome sequencing results of 12 ESCC patients revealed that $21 \%$ of patients had Notch1-inactivating mutations (24). This indicated that the Notch1 signal intrinsically exerts tumor-suppressive effects. However, a study of 60 patients with ESCC, 42 patients with benign esophageal squamous epithelium, and 13 patients with eosinophilic esophagitis with continuous NICD immunohistochemical staining showed that patients with ESCC had high NICD expression and were more likely to 


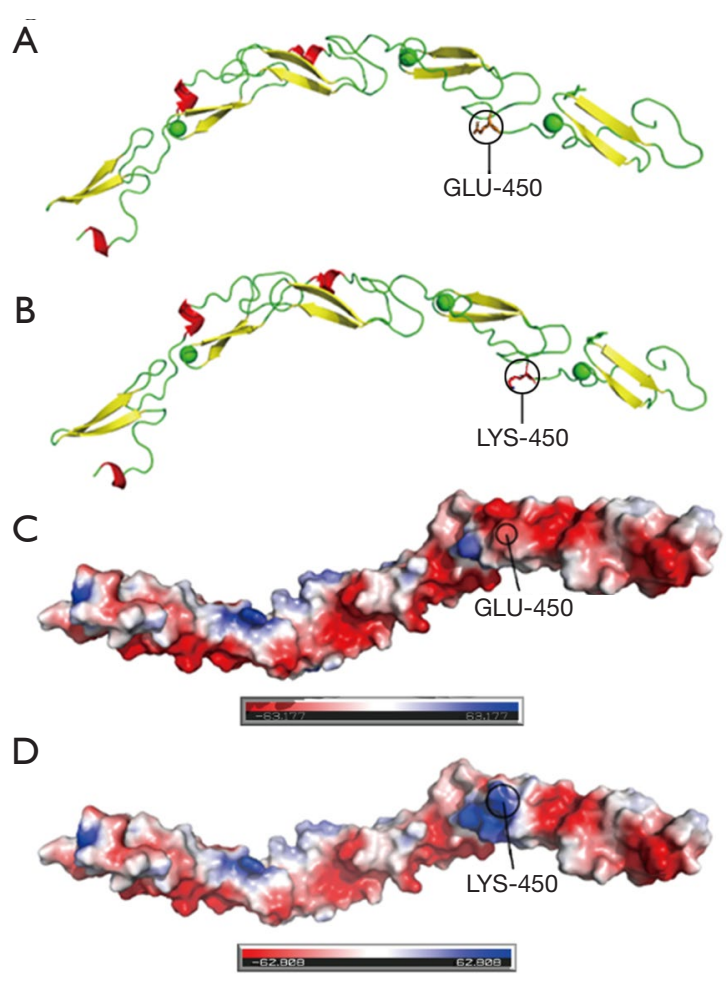

Figure 3 Three-dimensional structure of the EGF domain of Notch1 protein (298-488AA). (A and C) are the structural diagrams of wild-type proteins, and (B and D) are the structural diagrams of site-specific mutations using PyMOL; (A and B) are protein structures shown in cartoon form, in which the red structure is an alpha helix, the yellow structure is a $\beta$-pleated sheet, the green bar is a loop, and the green ball is a Ca ion; (C and D) are schematic diagrams of the protein surface charge (blue represents positive charge, red represents negative charge, and white represents neutral charge), and the surface charge at amino acid 450 of the mutant changes from negative to positive.

die from the disease (25), and another study of the Notch1 signaling pathway in EC-9706 cells involving the induction of epithelial-to-mesenchymal transition (EMT) through Snail demonstrated that inhibiting Notch1 signaling can suppress tumor invasion and metastasis (26). This indicated that the Notch1 signal intrinsically exerts oncogenic effects. We believe that the differences in research conclusions are due to different research methods and small sample sizes, which further indicates the complexity of the Notch1 signaling pathway in esophageal cancer.

Chemotherapy resistance significantly reduces the survival of patients with ESCC. The results of some studies confirmed that abnormal activation of Notch1 signaling could lead to drug resistance in various tumors during chemotherapy, which was consistent with our previous studies. Alterations in microRNAs (miRNAs) are involved in the initiation and progression of human diseases, including cancer (27). Several studies have revealed that high expression of miRNAs can inhibit the Notch1 signaling pathway, thus enhancing the chemotherapy sensitivity of ovarian cancer (28), breast cancer (29), colorectal cancer (30) and prostate cancer (31). Several studies have shown that activation of the Notch1 signaling pathway can enhance the chemotherapeutic resistance of cancer cells (32-37), including esophageal cancer cells (33). In general, the resistance of tumor cells to chemotherapy was increased by upstream signaling or the direct activation of the Notch1 signaling pathway.

We previously found that Notch 1 mutations might be associated with NAC resistance in patients with ESCC through NGS (6). The results of the present study demonstrated that the mutation site was located in the Notch1-DLL4 binding site, and the mutant domain was found to be highly conserved through bioinformatics analysis, highlighting the importance of this mutation site. By further analysis, we found that the mutation changed the protein surface charge of the binding site and increased the interaction force of the noncovalent bond compared with that of the wild type protein. This change might activate the Notch1 signaling pathway, leading to NAC resistance. However, one methodology limitation of this study was the lack of external validation in real-world samples from patients with ESCC and Notch1 signaling pathway alterations. Analyzing such samples is important and necessary for the study of the mechanism of NAC resistance in patients with ESCC, and we will perform these analyses in the future.

\section{Conclusions}

Missense mutations in the Notch1 gene lead to changes in the receptor surface charge and an increase in the number of noncovalent bonds in the binding site, which may cause the Notch1 signaling pathway to become more active. This may lead to NAC resistance in patients with ESCC. However, further in vitro experiments are needed to confirm our hypothesis. 
A
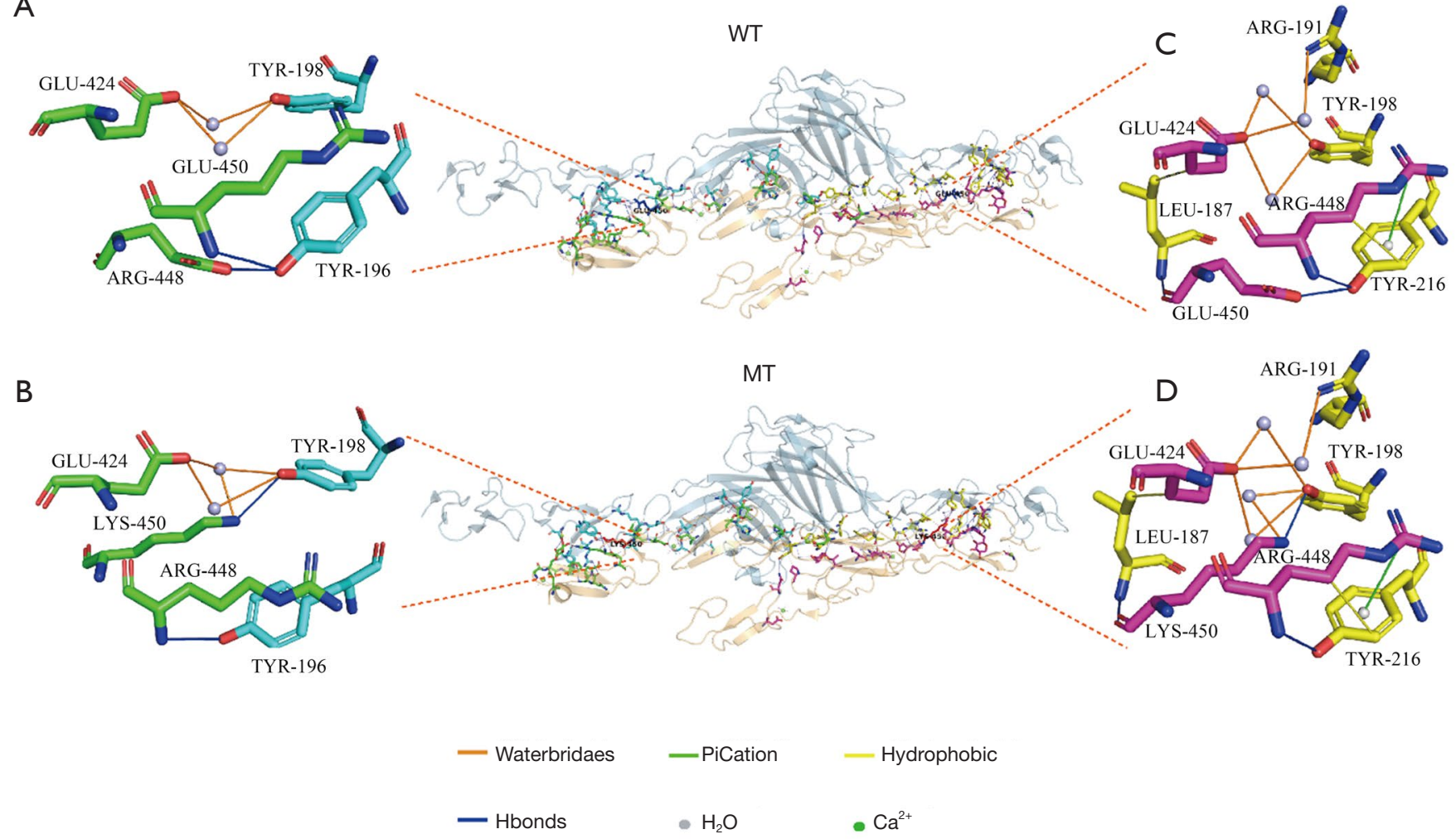

Figure 4 Interaction analysis between Notch1 and DLL4. (A, B, C and D) show the interaction of amino acid residues around the 450th amino acid (p.E450) WT and MT, respectively; the interaction between Notch1-DLL4 complex A chain (Notch1) and DLL4 is shown on the left (A and B) and the interaction between Notch1-DLL4 complex D chain (Notch1) and DLL4 is shown on the right (C and D) because the Notch1 protein is dimer; the green bar structure represents the A-chain amino acid residue (Notch1), and the cyan bar structure represents the DLL4 amino acid residue; the purple bar structure represents the D-chain amino acid residue (Notch1), and the yellow bar structure represents the DLL4 amino acid residue. WT, wild type; MT, mutation type.

Table 1 Noncovalent interactions in the Notch1-DLL4 complex WT and MT

\begin{tabular}{|c|c|c|c|c|c|c|}
\hline Genetic types & \multicolumn{2}{|c|}{ A chain } & \multicolumn{4}{|c|}{ D chain } \\
\hline WT & 2 & 2 & 3 & 3 & 2 & 1 \\
\hline MT & 3 & 2 & 4 & 3 & 2 & 1 \\
\hline
\end{tabular}

WT, wild type; MT, mutation type.

\section{Acknowledgments}

Funding: This work was supported by the National Natural Science Foundation of China (No. 82002521) and Natural Science Foundation of Henan Province (No. 202300410389).

\section{Footnote}

Conflicts of Interest: All authors have completed the ICMJE uniform disclosure form (available at https://dx.doi. org/10.21037/tcr-21-447). The authors have no conflicts of interest to declare. 
Ethical Statement: The authors are accountable for all aspects of the work in ensuring that questions related to the accuracy or integrity of any part of the work are appropriately investigated and resolved. The study was conducted in accordance with the Declaration of Helsinki (as revised in 2013). Institutional ethical approval was waived.

Open Access Statement: This is an Open Access article distributed in accordance with the Creative Commons Attribution-NonCommercial-NoDerivs 4.0 International License (CC BY-NC-ND 4.0), which permits the noncommercial replication and distribution of the article with the strict proviso that no changes or edits are made and the original work is properly cited (including links to both the formal publication through the relevant DOI and the license). See: https://creativecommons.org/licenses/by-nc-nd/4.0/.

\section{References}

1. Ando N, Kato H, Igaki H, et al. A randomized trial comparing postoperative adjuvant chemotherapy with cisplatin and 5 -fluorouracil versus preoperative chemotherapy for localized advanced squamous cell carcinoma of the thoracic esophagus (JCOG9907). Ann Surg Oncol 2012;19:68-74.

2. Zheng Y, Li Y, Liu X, et al. Reevaluation of Neoadjuvant Chemotherapy for Esophageal Squamous Cell Carcinoma: A Meta-Analysis of Randomized Controlled Trials Over the Past 20 Years. Medicine (Baltimore) 2015;94:e1102.

3. Shen LY, Wang H, Dong B, et al. Possible prediction of the response of esophageal squamous cell carcinoma to neoadjuvant chemotherapy based on gene expression profiling. Oncotarget 2016;7:4531-41.

4. Zhao WS, Yan WP, Chen DB, et al. Genome-scale CRISPR activation screening identifies a role of ELAVL2CDKN1A axis in paclitaxel resist ance in esophageal squamous cell carcinoma. Am J Cancer Res 2019;9:1183200.

5. Ooizumi Y, Kojima K, Igarashi K, et al. Comprehensive Exploration to Identify Predictive DNA Markers of DeltaNp63/SOX2 in Drug Resistance in Human Esophageal Squamous Cell Carcinoma. Ann Surg Oncol 2019;26:4814-25.

6. Liu J, Xing W, Tian Q, et al. Application of nextgeneration sequencing in resistance genes of neoadjuvant chemotherapy for esophageal cancer. Translational Cancer Research 2020;9:4847-56.

7. Bray SJ. Notch signalling in context. Nat Rev Mol Cell
Biol 2016;17:722-35.

8. Meurette O, Mehlen P. Notch Signaling in the Tumor Microenvironment. Cancer Cell 2018;34:536-48.

9. Fabbri G, Dalla-Favera R. The molecular pathogenesis of chronic lymphocytic leukaemia. Nat Rev Cancer 2016;16:145-62.

10. De Bie J, Demeyer S, Alberti-Servera L, et al. Single-cell sequencing reveals the origin and the order of mutation acquisition in T-cell acute lymphoblastic leukemia. Leukemia 2018;32:1358-69.

11. Karube K, Enjuanes A, Dlouhy I, et al. Integrating genomic alterations in diffuse large B-cell lymphoma identifies new relevant pathways and potential therapeutic targets. Leukemia 2018;32:675-84.

12. Kridel R, Meissner B, Rogic S, et al. Whole transcriptome sequencing reveals recurrent NOTCH1 mutations in mantle cell lymphoma. Blood 2012;119:1963-71.

13. Westhoff B, Colaluca IN, D'Ario G, et al. Alterations of the Notch pathway in lung cancer. Proc Natl Acad Sci U S A 2009;106:22293-8.

14. Wang K, Zhang Q, Li D, et al. PEST domain mutations in Notch receptors comprise an oncogenic driver segment in triple-negative breast cancer sensitive to a gammasecretase inhibitor. Clin Cancer Res 2015;21:1487-96.

15. Martincorena I, Fowler JC, Wabik A, et al. Somatic mutant clones colonize the human esophagus with age. Science 2018;362:911-7.

16. Ogawa R, Ishiguro H, Kimura M, et al. NOTCH1 expression predicts patient prognosis in esophageal squamous cell cancer. Eur Surg Res 2013;51:101-7.

17. Luca VC, Jude KM, Pierce NW, et al. Structural biology. Structural basis for Notch1 engagement of Delta-like 4. Science 2015;347:847-53.

18. Kojika S, Griffin JD. Notch receptors and hematopoiesis. Experimental Hematology 2001;29:1041-52.

19. Radtke F, Schweisguth F, Pear W. The Notch 'gospel'. EMBO Rep 2005;6:1120-5.

20. Agrawal N, Frederick MJ, Pickering CR, et al. Exome sequencing of head and neck squamous cell carcinoma reveals inactivating mutations in NOTCH1. Science 2011;333:1154-7.

21. Stransky N, Egloff AM, Tward AD, et al. The mutational landscape of head and neck squamous cell carcinoma. Science 2011;333:1157-60.

22. Wang NJ, Sanborn Z, Arnett KL, et al. Loss-of-function mutations in Notch receptors in cutaneous and lung squamous cell carcinoma. Proc Natl Acad Sci U S A 2011;108:17761-6. 
23. George J, Lim JS, Jang SJ, et al. Comprehensive genomic profiles of small cell lung cancer. Nature 2015;524:47-53.

24. Agrawal N, Jiao Y, Bettegowda C, et al. Comparative genomic analysis of esophageal adenocarcinoma and squamous cell carcinoma. Cancer Discov 2012;2:899-905.

25. Lubin DJ, Mick R, Shroff SG, et al. The notch pathway is activated in neoplastic progression in esophageal squamous cell carcinoma. Hum Pathol 2018;72:66-70.

26. Wang T, Xuan X, Pian L, et al. Notch-1-mediated esophageal carcinoma EC-9706 cell invasion and metastasis by inducing epithelial-mesenchymal transition through Snail. Tumour Biol 2014;35:1193-201.

27. Calin GA, Croce CM. MicroRNA signatures in human cancers. Nat Rev Cancer 2006;6:857-66.

28. Zhou Y, Chen Q, Qin R, et al. MicroRNA-449a reduces cell survival and enhances cisplatin-induced cytotoxicity via downregulation of NOTCH1 in ovarian cancer cells. Tumour Biol 2014;35:12369-78.

29. Park EY, Chang E, Lee EJ, et al. Targeting of miR34aNOTCH1 axis reduced breast cancer stemness and chemoresistance. Cancer Res 2014;74:7573-82.

30. Liu H, Yin Y, Hu Y, et al. miR-139-5p sensitizes colorectal cancer cells to 5 -fluorouracil by targeting NOTCH-1. Pathol Res Pract 2016;212:643-9.

31. Liu X, Luo X, Wu Y, et al. MicroRNA-34a Attenuates

Cite this article as: Gao K, Xing W, Liu X, Liu J, Sun H, Hao W, Zheng Y. The Notch1 gene may control cell chemoresistance in esophageal squamous cell cancer. Transl Cancer Res 2021;10(7):3278-3285. doi: 10.21037/tcr-21-447
Paclitaxel Resistance in Prostate Cancer Cells via Direct Suppression of JAG1/Notch1 Axis. Cell Physiol Biochem 2018;50:261-76.

32. Ye QF, Zhang YC, Peng XQ, et al. Silencing Notch-1 induces apoptosis and increases the chemosensitivity of prostate cancer cells to docetaxel through Bcl-2 and Bax. Oncol Lett 2012;3:879-84.

33. Subramaniam D, Ponnurangam S, Ramamoorthy P, et al. Curcumin induces cell death in esophageal cancer cells through modulating Notch signaling. PLoS One 2012;7:e30590.

34. Liu C, Li Z, Bi L, et al. NOTCH1 signaling promotes chemoresistance via regulating ABCC1 expression in prostate cancer stem cells. Mol Cell Biochem 2014;393:265-70.

35. Hang Q, Sun R, Jiang C, et al. Notch 1 promotes cisplatinresistant gastric cancer formation by upregulating lncRNA AK022798 expression. Anticancer Drugs 2015;26:632-40.

36. Hassan WA, Yoshida R, Kudoh S, et al. Notch1 controls cell chemoresistance in small cell lung carcinoma cells. Thorac Cancer 2016;7:123-8.

37. Zeng, Liang YK, Xiao YS, et al. Inhibition of Notch1 reverses EMT and chemoresistance to cisplatin via direct downregulation of MCAM in triple-negative breast cancer cells. Int J Cancer 2020;147:490-504. 